\title{
Spinal Angiomas as a Cause of Non-traumatic Paraplegia: Case Report
}

\author{
R. Chappel, MD, ${ }^{1}$ A. M. de Barsy, $M D,{ }^{4}$ G. Dua, $M D,{ }^{2}$ B. Appel, $M D,{ }^{3}$ \\ P. Herregods, MD $^{1}$ \\ ${ }^{1}$ Department of Physical medicine and rehabilitation ${ }^{2}$ Department of neurosurgery \\ ${ }^{3}$ Department of Neuroradiology, Middelheimziekenhuis, Antwerp ${ }^{4}$ Department of \\ Neurology, Vesaliusziekenhuis, Brasschaat, Belgium.
}

\begin{abstract}
Summary
A case is presented of a 17-year-old boy who developed paraplegia after a sudden low back pain, like a 'stab in the back', with radicular radiation into the lower limbs. No trauma preceded the onset of symptoms.

The diagnosis was finally made by neuroradiological investigations (magnetic resonance imaging (MRI), myelography and $C T$-scan).

$A$ review of the physiopathology of spinal angiomas, extra and/or intramedullary, by exclusion of vertebral (haem) angiomas, is made.
\end{abstract}

Key words: Spinal angioma; $M R I ;$ Myelography; CT-scan; Spinal angiography; Paraplegia.

Spinal angiomas as a part of spinal cord arteriovenous malformations (AVM), which means an abnormal arteriovenous communication permitting the arterial blood to enter the venous system without passing through a capillary network, constitute 3.3 to $11 \%$ of spinal cord tumours (Ommaya, 1973; Aminoff, 1976; Yasargil, 1984). Although a sudden or progressive myelopathy before or after treatment has been mentioned in many publications, no exact figures of the occurrence of permanent paraplegia are reported.

The diagnosis is usually made by myelography followed by spinal angiography with selective arteriography (Di Chiro, 1967; Doppman, 1969). Recently more information can be obtained by CT-scan and MRI (Di Chiro, Doppman et al., 1985). Although therapeutic measures such as excision of the anomaly, decompressive laminectomy, ligation of the arterial supply and percutaneous embolisation are proposed for different types of AVM (Ommaya, 1973; Yasargil, 1984), an unusual course of the disease and a rare localisation of the angioma may cause diagnostic and therapeutic problems, as are demonstrated in the following case. 


\section{Case report}

A 17-year-old boy was admitted to hospital on the 6th August 1986. On the preceding evening sudden severe back pain appeared with radicular radiation first to the left leg, and afterwards to the right. Rapidly thereafter progressive loss of motor and sensory functions in the lower limbs appeared. There was no illness, trauma or infection in the previous days.

On admission, the general physical examination was normal, except for a globus vesicalis. Neurological examination showed a flaccid paralysis of both legs with absent knee and ankle-jerks. A sinistroconvex dorsolumbar scoliosis, probably paralytic, with slight right thigh atrophy was previously noted.

Lumbar punctures on the first and second days (blood stained, with red blood cells), electromyography of the lower limbs, X-rays of the thoracic and thoracolumbar spine and myelography and CT-scan (from T9 to L4) performed at a peripheral hospital, were not helpful. As no change occurred in patient's condition, he was referred to our hospital for MRI and a tumour like process from T9-T10 to the conus terminalis (Figure 1) was demonstrated.

A bone scan was normal. Further myelography now revealed a block at the T11-T12 level, and now CT-scan suggested an intramedullary malformation (Figure 2) from the middle of T11 to the level T11-T12; a changing and increased density in the malformation and the partial absence of contrast filling of the subarachnoid space at a lower level suggested an angioma fed at the right anterior side of the spinal cord (Figure 3). A laminectomy from T9 to T12 was performed on the 13th of August with partial resection of an intramedullary AVM and drainage of a caudally situated collection of blood.

Unfortunately no improvement in the patients neurological state occurred, and about 1 month later spinal angiography was performed. By selective catheterisation of the left L2 artery a pathological dilated anterior spinal artery with a branch draining directly into an intramedullary AVM at T10 situated in the right hemimyelum (Figure 4) was shown. Three vascular dilatations seem to compose the 'nidus'. The angioma drains very quickly into a dilated superficial vein. This leads to a marked 'steal phenomena'. Because the origin of the spinal artery was very close to a lumbar artery no further treatment (embolisation) was carried out in order to avoid peripheral arterial embolisation of the lower limbs. Furthermore the chance of recovery had become very minimal after the prolonged spinal cord damage.

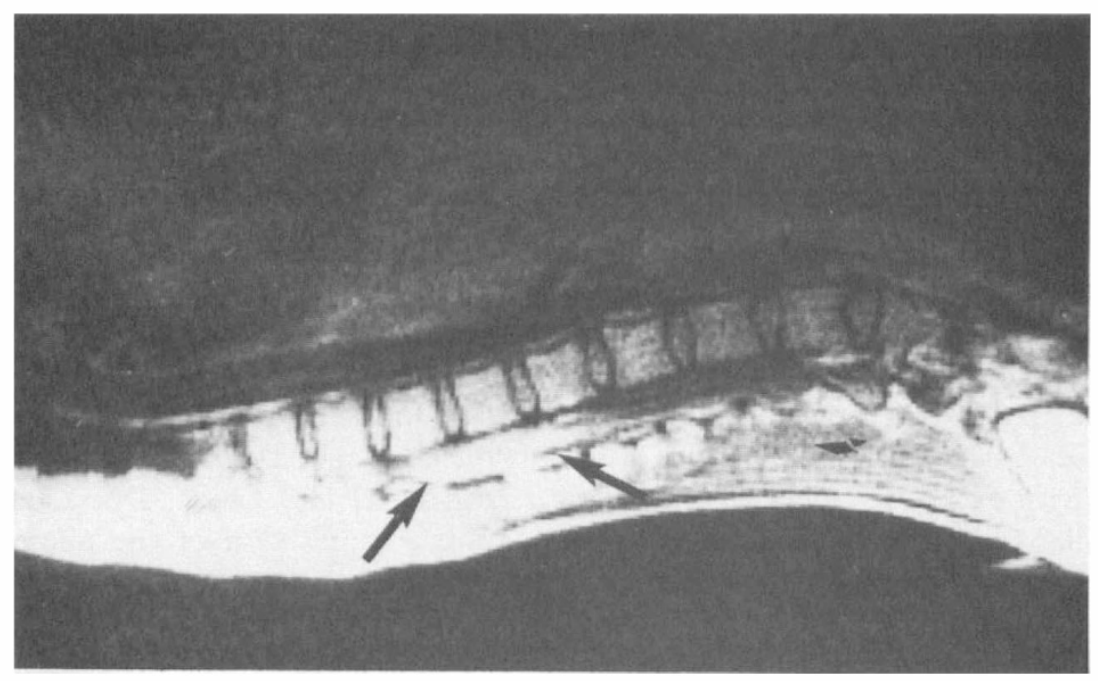

Figure 1 MRI: the arrows show a tumourlike process from the level T9-T10 to the conus terminalis. 


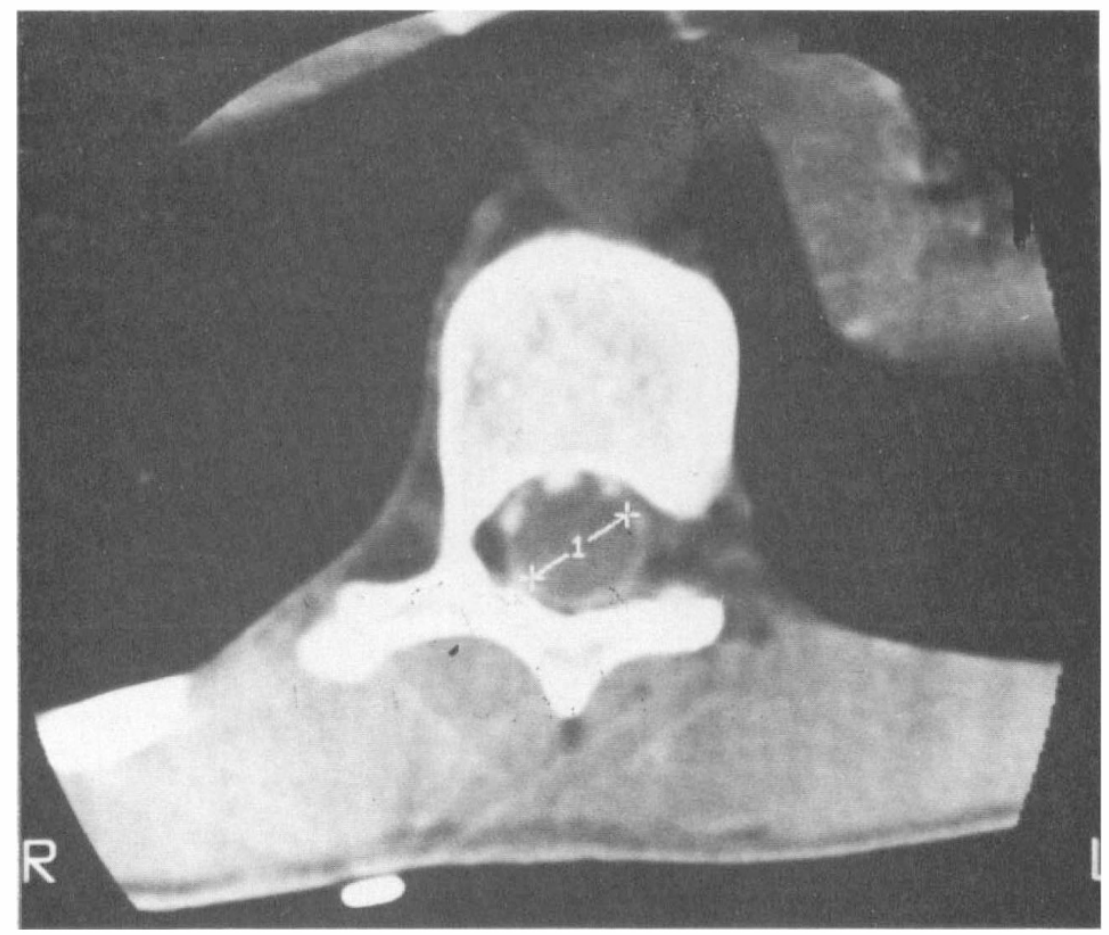

Figure $2 \mathrm{CT}$-scan showing a significant widening of the medulla at the level T10.

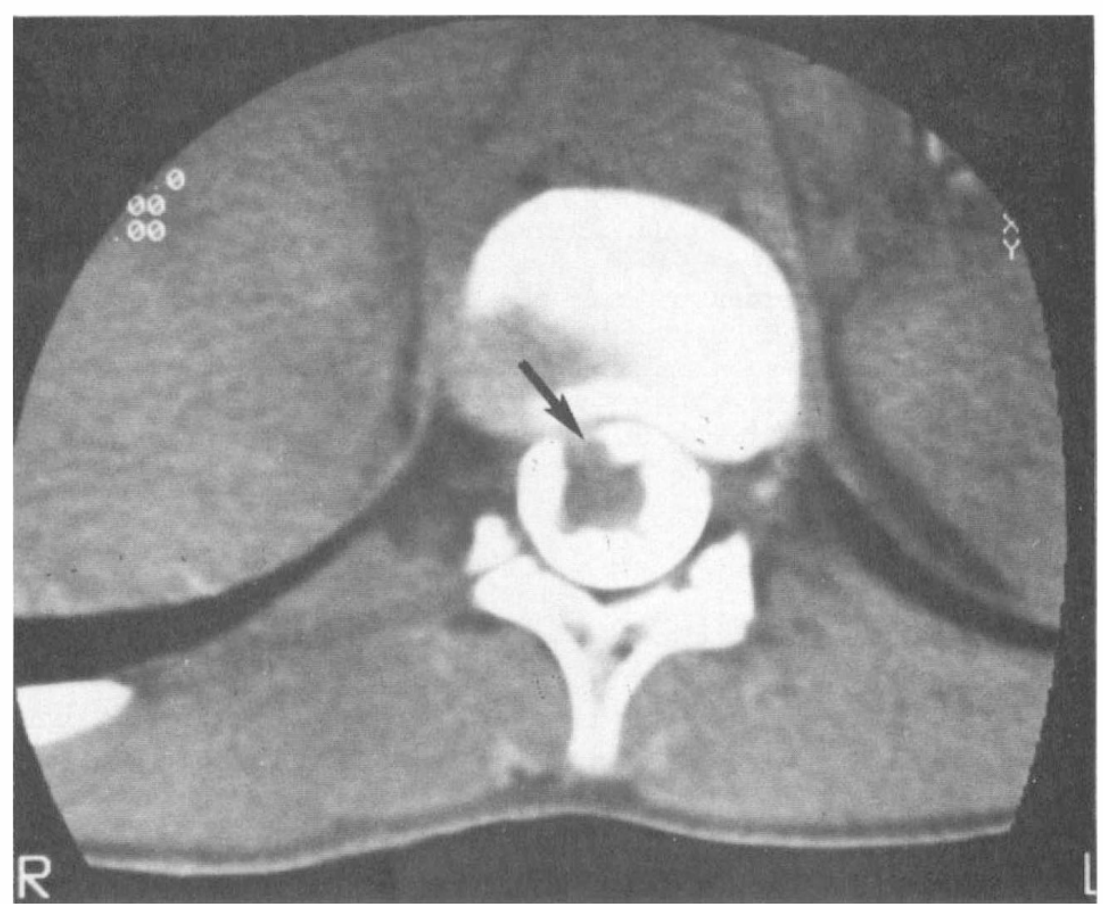

Figure 3 CT-scan at the level T11: defect of contrast filling of the subarachnoid spaces at the right anterior side (arrow), corresponding to an anterior artery supply of the angioma. 


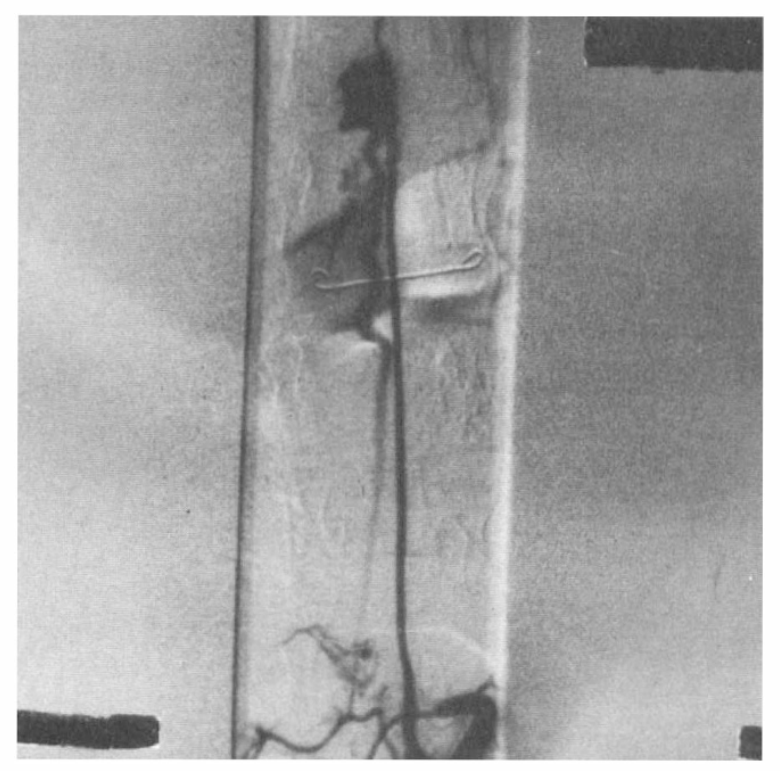

Figure 4 Angiography with selective catheterisation of the left L 2 artery shows a pathological spinal anterior artery, the AVM (level T10) draining directly into a dilated vein and the marked 'steal phenomena'.

Complete flaccid paraplegia persisted with loss of urinary and bowel control. Full rehabilitation was started. It is remarkable that a similar history was obtained in our patient 8 years before; after a nocturnal appearance of sudden low back pain, paraparesis with a sensory level at $\mathrm{L} 1$ with urinary retention occurred. Two weeks later progressive recovery started with full recovery after 2 months.

The following examinations were done at that time: normal blood examinations with only a slight elevated sedimentation rate; repeated lumbar punctures with initially increased number of RBC $\left(300-400 / \mathrm{mm}^{3}\right)$, without marked elevation of the protein count or positive cultures. Myelography 1 day after hospitalisation was interpreted as being normal, although post hoc some widening of the cord and tortuous vessels, probably enlarged veins were visible. Repeated electromyography examinations revealed no significant signs of denervation or conduction velocity changes in the lower limbs, although for the distal latencies some slowing was noted. Because of the spontaneous recuperation and the lack of further diagnostic investigations, a tentative diagnosis of a Guillain Barré syndrome was made.

\section{Discussion}

Considering the embryology of the spinal cord blood supply, composed of a primitive radicular capillary network on the surface of the cord, finally one anterior and two posterior spinal arteries are formed; with maturation, the adult vascular supply, which emphasises a few dominant feeding arteries, is established (Ommaya, 1973; Yasargil, 1984). One of the most common hypotheses for the existence of an AVM is the persistence of portions of the primitive AV network especially on the dorsal surface of the cord.

About $70 \%$ of the patients, said to be 2 to 3 times more common in males, are situated below T9 (Aminoff, 1976), usually extramedullary, and in 
$80 \%$ situated posterior to the cord (Djindjian, 1972); mainly or completely intramedullary localisation of angiomas was thought to be rather rare, $8 \%$ (Djindjian, 1969), but more recent publications (Cogen, 1983; Hurt, 1978) report a significant intramedullary component in about $35 \%$ to $60 \%$ of the cases.

The diagnosis of spinal angiomas, especially in patients with a sudden or progressive myelopathy remains very important. Initial symptoms such as lower back pain, followed by sensory disturbances preceding progressive motor weakness in the lower limbs, are common. The classic described, 'stab in the back', suggesting a subarachnoid haemorrhage is rare. Technical investigations for accurate diagnosis are essentially radiological; plain $\mathrm{X}$-rays of the spine are seldom of value; essential are myelography followed by spinal angiography with selective arteriography as described in the reports of Di Chiro (1967) and Doppman (1969). CT-scan and MRI are additional non-invasive techniques which can offer some advantages. A CT-scan can measure the extent of the lesion and density changes in the cord, MRI can facilitate discrimination between intra-and extramedullary lesions, including the nidus of an AVM (Di Chiro, 1985). However, both cannot replace the essential investigations till now. Based on selective angiography a classification into three types of AVM has been proposed (Ommaya, 1973): the single coiled vessel anomaly (type I), the glomus anomaly (type II) and the 'juvenile' anomaly (type III); this type is predominantly found in children and young adults, comprises 10 to $15 \%$ of the vascular malformations of the spinal cord and can be found intramedullary; the lesion can be fed by many arteries with a rapid flow of contrast medium and is not restricted to the dorsal aspect of the cord.

The importance, implications and resectability of intramedullary AVM have been stressed by different authors (Djindjian, 1969; Owen, 1979; Cogen, 1983; Yasargil, 1984).

Supplying the angioma by the anterior spinal artery or by a branch of it seems exceptional unless in the cervical region (Djindjian, 1972; Ommaya, 1973; Aminoff, 1976). Thoracolumbar AVM with intramedullary components and anterior feeding vessels are reported by Cogen (1983) and by Yasargil (1984), who reported $24 \%$ of entirely intramedullary lesions half in the cervical and half in the thoracolumbar region; the cause of sudden neurological deterioration was attributed to subarachnoid haemorrhage (SAH) or intramedullary haemorrhage (IMH), or to thrombosis within the venous varices associated with the AVM.

Concerning the patient that we present, we can put forward a rather rare case of an anterior, intramedullary angioma, situated at level T10 fed directly by a branch of the anterior spinal artery. Pathophysiologically a diminished intramedullary AV pressure gradient with ischaemia of the spinal cord, subarachnoid haemorrhages, intravascular thrombosis, steal phenomena with diversion of blood from the spinal cord to the angioma and even cord compression by enlarged pial vessels are factors in favour to the clinical neurological picture (Aminoff, 1976). Symptoms of spinal angiomas may be precipitated or aggravated by exercise, systemic infection, unrelated trauma and menstruation (Pia, 1973). Our patient was a sporting man and recently an amateur boxer. The history of our patient can probably be explained by episodes of intramedullary 
and subarachnoid haemorrhages with spontaneous resorption and recovery of the paraparesis at the first observation, other mechanisms are not excluded here, and with definite damage to the cord at the second occasion. As shown on post-operative angiography, there is certainly a factor of steal phenomena influencing the clinicopathological picture.

Although embolisation and intramedullary micro-surgery are the techniques of choice (Cogen, 1983; Yasargil, 1984), the difficulty of totally removing intramedullary lesions without jeopardising spinal cord function is also established (Krayenbühl, 1969).

On the other hand it is important to state that in cases of non-traumatic paraplegia with rapid neurological deterioration, MRI can complement myelography and CT-scan in detecting spinal angiomas, before angiography is performed, especially in children and in young adults.

\section{References}

Aminoff MJ 1976 Spinal angiomas, Blackwell Scientific Publications Ch. 10, pp. 82-95; Ch. 13, pp. 131-136.

COGEN P, STEIN BM 1983 Spinal cord arteriovenous malformations with significant intramedullary components. Fournal of Neurosurgery 59:471-478.

Di Chiro G, Doppman J, OMMAYA AK 1967 Selective arteriography of arteriovenous aneurysms of spinal cord. Radiology 88:1056-1077.

Di Chiro G, Doppman JL et al. 1985 Tumors and arteriovenous malformation of the spinal cord: assessment using MR. Radiology, September 1985:689-697.

DJINDJIAN R 1972 Neuroradiological examinations of spinal cord angiomas. Handbook of clinical Neurology vol. 12, 631-643.

Doppman JL, Di Chiro G, Ommaya AK 1969 Selective arteriography of the spinal cord, St. Louis, Mo., Warren Green, Inc.

HURT M, Houdart R, DJINDJian R et al. 1978 Arteriovenous malformations of the spinal cord. Clinical, anatomical and therapeutic considerations: a series of 150 cases Progressive Neurological Surgery 9:238-266.

KRAYENBÜHL M, YASARGIL MG, MCClintock HG 1969 Treatment of spinal cord vascular malformations by surgical excision. Fournal of Neurosurgery 30:436-445.

OMMAYA AY 1973 Arteriovenous malformations of the spinal cord. In: Neurological surgery, pp. 852-862. Julian R. Youmans (ed). WB Sanders Company, Philadelphia, London, Toronto.

OWEN MP, Brown RH, SPETZLER RF et al. 1979 Excision of intramedullary arteriovenous malformations using intraoperative spinal cord monitoring Surgical Neurology 12:271-277.

PIA HW 1973 Diagnosis and treatment of spinal angiomas Acta neurochirurgica 28:1-12.

YASARGIL MG, SYMON L and TEDDY PJ 1984 Arteriovenous malformations of the Spinal Cord. In: Advances and Technical Standards in Neurosurgery. Springer-Verlag, Wien, New York, Vol. 11, pp. 62-99. 